\title{
Chronic encapsulated expanding hematoma after stereotactic radiosurgery of cerebral arteriovenous malformation
}

\author{
Mi-Sun Choi', Mee Joo' ${ }^{2}$, Chan-Young Choi ${ }^{3}$ \\ 'Department of Neurosurgery, Seoul Paik Hospital, College of Medicine, Inje University, ${ }^{3}$ Department of Neurosurgery \\ and 'Pathology, Ilsan Paik Hospital, College of Medicine, Inje University
}

Stereotactic radiosurgery has become excellent alternative treatment for cerebral arteriovenous malformations (AVM). This technique has expanded to treatment of larger AVM which is not amenable to surgical management. However, its variable adverse effects should be also taken into considerations sincerely because of radiobiological characteristics such as delayed onset and progressive neurological deteriorations. Herein, we report a case in which progressively expanding hemorrhagic cyst with repeated bleedings so called chronic encapsulated expanding hematoma was developed on several years after radiosurgery treatment. Neurological and radiological findings were improved by surgical removal.

$J$ Cerebrovasc Endovasc Neurosurg. 2019 September;21(3):152-157

Received : 26 July 2019

Revised : 18 September 2019

Accepted : 28 September 2019

Correspondence to Chan Young Choi Department of Neurosurgery, Ilsanpaik

hospital, Inje University

\author{
Tel : 82-2-2270-0029 \\ Fax : 82-2-2270-0294 \\ E-mail : cychoi@paik.ac.kr \\ ORCID : http://orcid.org/0000-0003-4750-7661
}

Keywords chronic encapsulated expanding hematoma, adverse radiation effect, radiosurgery, arteriovenous malformation

This is an Open Access article distributed under the terms of the Creative Commons Attribution NonCommercial License /http://creativecommons.org/licomm/by-nc/3.0) which permits unrestricted nonmedium, provided the original work is properly cited.

\section{INTRODUCTION}

Stereotactic radiosurgery (SRS) has become a widely used and accepted alternative treatment modality for cerebral arteriovenous malformation (AVM) because of its excellent clinical outcomes. Recently, this treatment modality and advanced techniques has contributed to the management of larger AVMs which are difficult to surgery ${ }^{1319)}$. However, several adverse radiation effects with delayed onset of SRS including radiation induced brain edema, cyst formation, radiation necrosis, dural arteriovenous fistula, chronic encapsulated expanding hematoma, radiation induced tumor, radiation induced cavernoma have been reported ${ }^{3 / 6) 71017720)}$. Herein, we report a case that growing cystic mass along with multiple minor intra- cerebral bleedings was managed by surgical removal and review the relevant literatures.

\section{CASE REPORT}

A 46-year-old male patient presented with intermittent and non-specific headache for several weeks. Any specific neurological deficits were not found on neurological examinations. Brain computed tomography (CT) showed curvilinear structures with high density in the right occipital lobe which suggested a vascular lesion (Fig. 1). Magnetic resonance image (MRI) showed multiple round and linear signal voids with variable width and length were collected and its diameter was about $3 \mathrm{~cm}$ (Fig. 2). These radiological findings suggested an unruptured AVM. On the cere- 


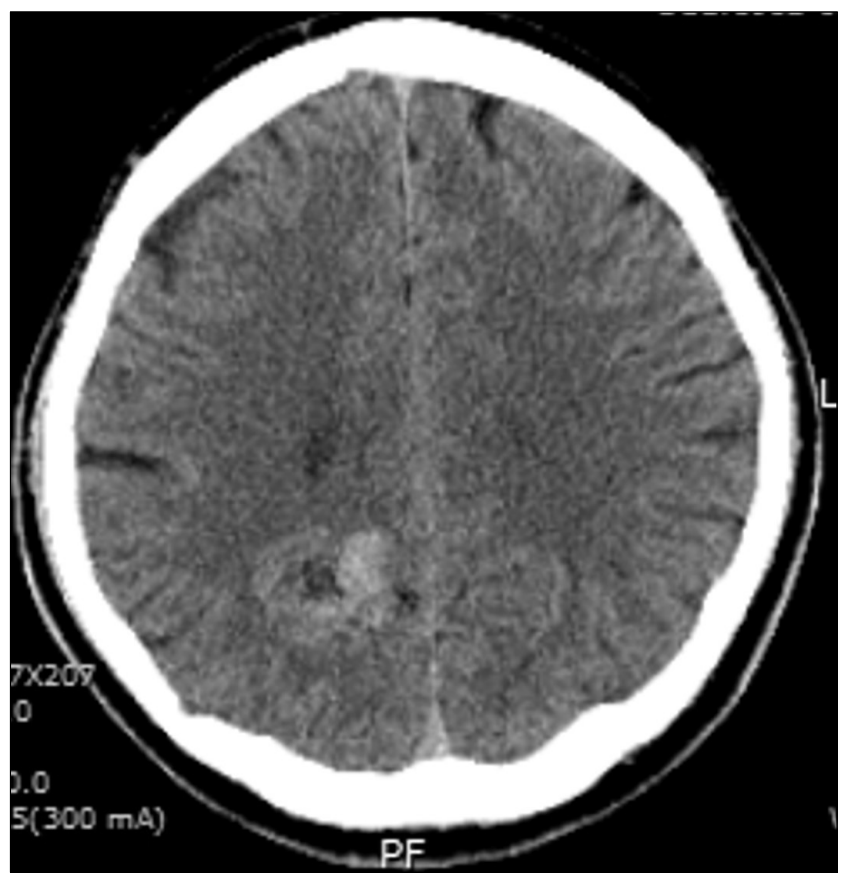

Fig. 1. Brain computed tomography shows a collection of curvilinear structures with high density in the right occipital lobe.

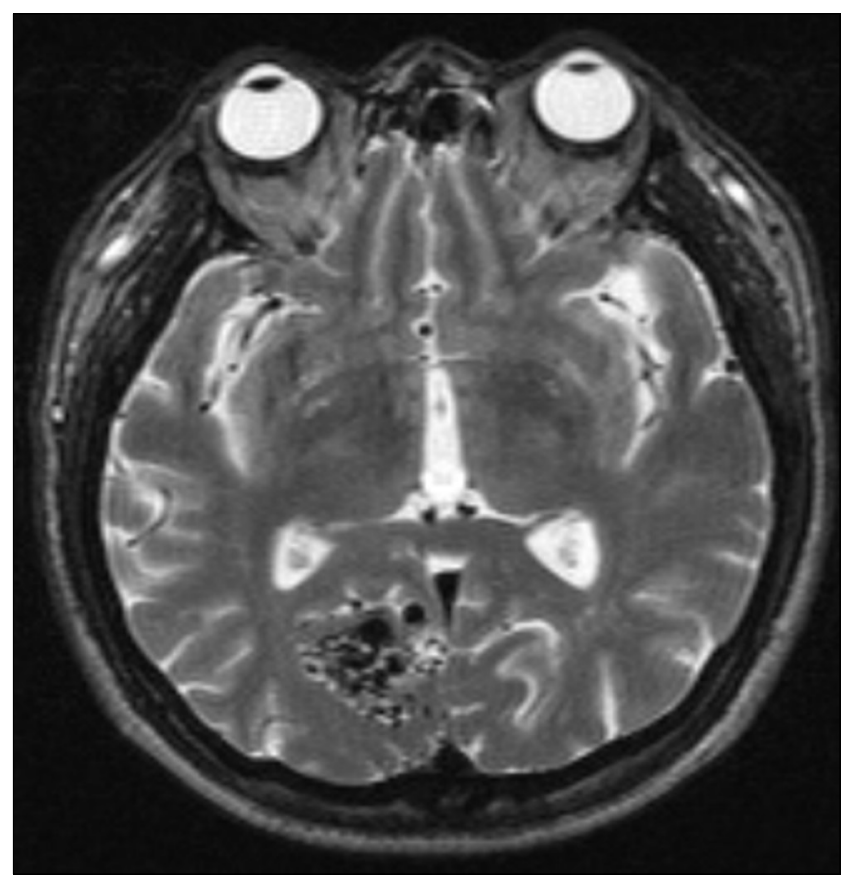

Fig. 2. Brain magnetic resonance imaging reveals multiple signal voids in the mass like lesion that have variable width, length, and size. These findings are suggesting vascular malformations.

bral angiograms, this AVM having a nidus of diffuse type had several arterial feeders supplied by distal branches of posterior cerebral artery (PCA) and middle cerebral artery (MCA). It was drained through the several cortical veins and straight sinus (Fig. 3). It was not amenable to surgical treatment as Spetzler-Martin grade IV. Stereotactic radiosurgery was determined for obliteration of AVM nidus. The AVM nidus volume was about $14 \mathrm{~cm} 3$ and 23 Gy with $80 \%$ isodose line was delivered. Approximately 1 year later after SRS, the perilesional low density on the CT and high signal change on T2 weighted MRI were found without definite mass effects. At 28 months after SRS, he presented with left visual field defect and intermittent headache. The follow up brain MRI showed irregular peripheral enhancement surrounding the irradiated field, increase of perilesional high signal changes, and ipsilateral ventricular compression due to mass effects. The conservative management was tried on that time. Since then, multiple small non-enhancing cysts were growing gradually within the T2 high signal region adjacent to the enhancing lesion. Along with increased mass effects and enlargement of cysts, small enhancing mass adhered to the posterior falx in the left occipital lobe which was contralateral to the irradiation side was also developed. At 5.5 years after SRS, brain CT scan performed with sudden headache and visual disturbance showed hemorrhage around the cyst in the right parietal lobe, however AVM nidus was totally obliterated in the cerebral angiograms performed at this time. Cyst with mass effect could be controlled through the repeated aspirations of Ommaya reservoir inserted. At 7 years after SRS, gradually growing enhancing mass and adjacent hemorrhagic cysts with mass effects were found in the left occipital lobe (Fig. 4). Fluid-fluid level with different signal densities within the cyst suggested multiple hemorrhages with different stages. The surgical resection was decided because of concerns about blindness, severe headache and nausea, while enhancing lesion and cyst in the right occipital lobe has been under the control. Intraoperatively, well demarcated mass with fragile granulomatous tissue was prone to 


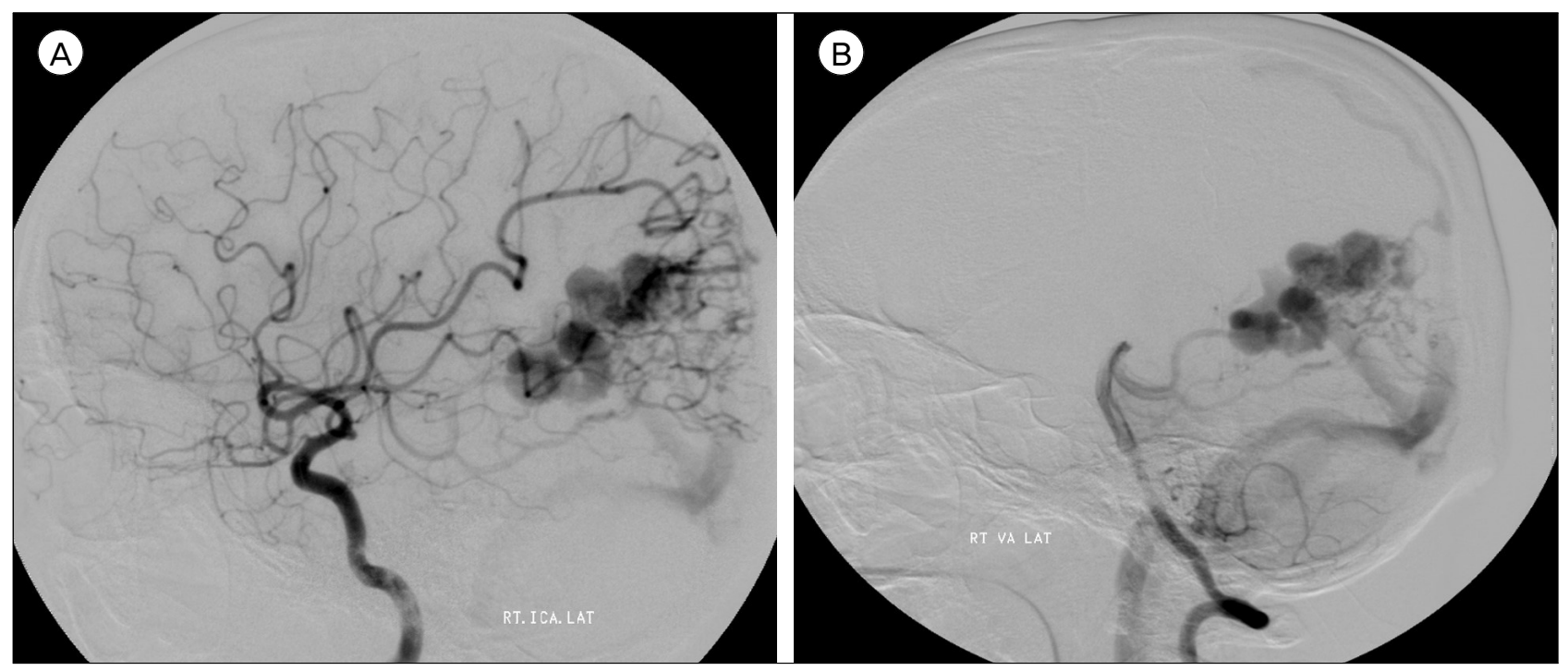

Fig. 3. On the lateral cerebral angiograms (A, B), the arteriovenous malformation has several feeders supplied by distal branches of middle cerebral artery and posterior cerebral artery, and has veins draining into the cortical veins and straight sinus.

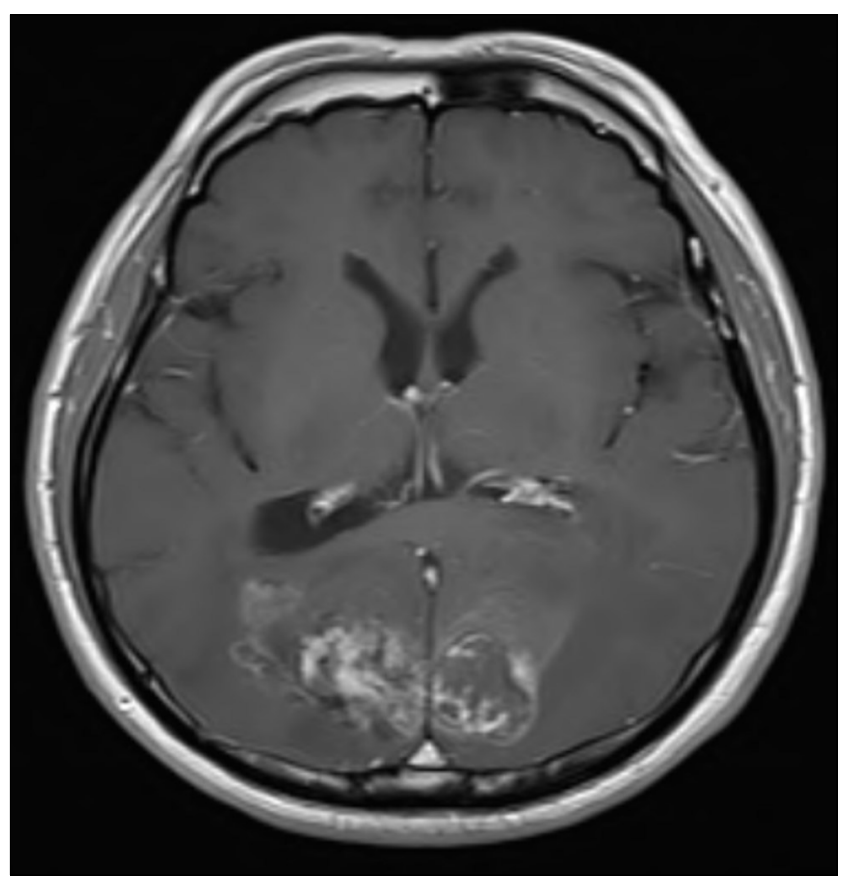

Fig. 4. At 7 years after stereotactic radiosurgery, magnetic resonance imaging shows multiple stages cerebral hemorrhages, including repeated bleedings and enhancing lesion adjacent to the falx in the left occipital lobe. The enhancing lesion with peripheral low signal rim has been grown while right enhancing lesion and cysts have been under the control by repeated aspiration through the omaya reservoir.

bleed and surrounded by old hemorrhages and gliotic tissues. His clinical symptoms were improved gradually after complete removal and preoperative visual field deficits were stable. The pathological findings were compatible to the chronic encapsulated hematoma which showed extensive hemorrhagic necrosis in the center and hemosiderin deposition in the peripheral fibrosis (Fig. 5).

\section{DISCUSSION}

The adverse effects of SRS have been well known. Among them, chronic encapsulated expanding hematoma can be experienced rarely although it has been reported with increasing frequency recently. And approximately $40 \%$ of reported cases are associated with vascular malformations, such as cavernoma, venous angioma and cerebral AVM. Generally, this entity has clinical characteristics of gradual onset and progressive enlargement. Hematoma enlargement with repeated local hemorrhages occur regardless of angiographic AVM obliteration. Our case was very similar to clinical findings of previous reports. However, the pathogenesis for developing chronic encapsulated expanding hematoma is still unknown ${ }^{14118)}$. Pathologically, it is organized hematoma surrounded by a tough capsule which is consisted of a fibrocollagenous outer layer and a granulating inner 


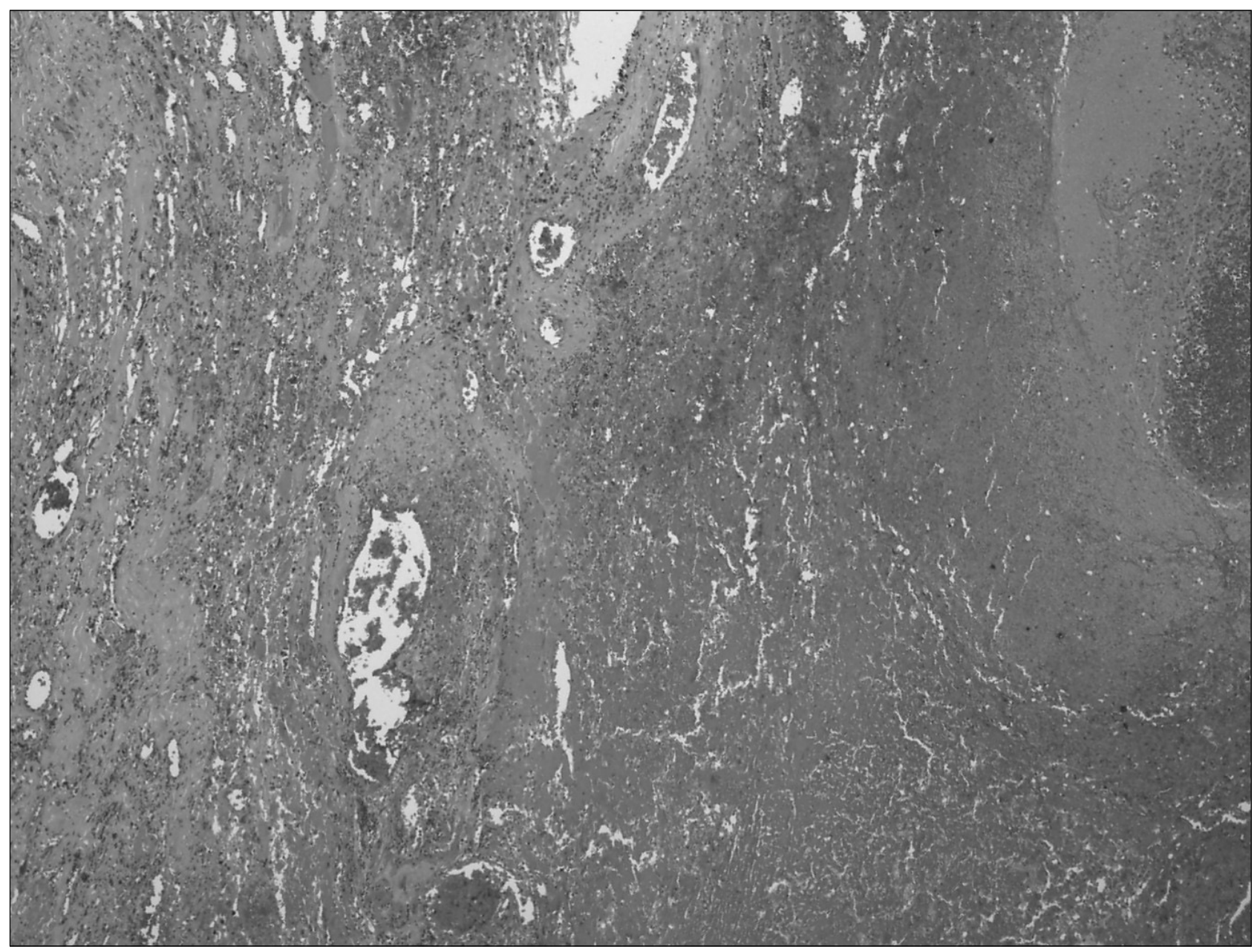

Fig. 5. Pathological findings present extensive hemorrhagic necrosis in the center and hemosiderin deposition in the peripheral fibrosis.

layer with prominent neovascularization and myofibroblast proliferation ${ }^{14)}$. The hematoma capsule may be formed by fibroblasts which are derived from neovascularized vessels and the hematoma expansion appears to enlarge by repeated focal hemorrhages or exudates from the fragile vessels formed within the inner layer of the hematoma capsule. Therefore, it is less likely that repeated hemorrhages occur from a degenerated, irradiated nidus. The nidus of AVM in our case was already obliterated completely on cerebral angiograms before repeated focal hemorrhages.

Vascular endothelial growth factor (VEGF) capable of modulating angiogenesis is expressed in the epithelial cells of the choroid plexus, astrocytes, neurons, granule cells, and fibroblasts and a specific regulator of endothelial cell growth and differentiation ${ }^{2111}$. It is also known as vascular permeability factor resulting in the development of cerebral edema by its ability to induce vascular leakage ${ }^{1)}$. During the process of AVM degeneration following radiosurgery, the nidus and surrounding cerebral tissues in the irradiated field are exposed to hypoxic state. This hypoxia is also considered to be the most effective force stimulating VEGF transcription ${ }^{12}$. Therefore, local increase of VEGF following irradiation may promote the neovascularization and prone to occur multiple focal bleedings resulting in the enlargement of hematoma and cerebral edema. In our case, the accompanied cerebral edema and focal hemorrhages were disappeared after resection of this expanding hematoma. On the other hand, the 
spontaneously resolving cases of chronic encapsulated expanding hematoma has been also reported ${ }^{4) 5}$. It is suggested that the other unknown factors related to the expansion of hematoma may exist. Cyst after radiosurgery for AVM is common complication with $1.6 \%$ of incidence and non-epithelium lined cavity which is classified as pseudocyst. Although the exact mechanism of cyst formation remains unknown, increased permeability of injured blood vessels, breakdown of the blood brain barrier, liquefaction of coagulation necrosis and radiation induced inflammation in the brain parenchyma around the irradiated nidus have been suggested ${ }^{17 / 21)}$. The extravasation from the injured vessels resulting in the cyst formation and granulomatous changes such as newly developed fragile vessels resulting in the chronic repeated hemorrhages may occur in the irradiated fields. And then the increased protein content and osmotic pressure caused by chronic repeated hemorrhages from the granulomatous lesions can lead to the cyst enlargement. The origin of angiomatous nodular lesion is more likely in the adjacent brain parenchyma around irradiated area because the angiomatous lesions are not specific for AVM and has no continuity with the irradiated area ${ }^{17)}$. So, if the hemorrhage from an angiomatous lesion spreads mainly into the brain parenchyma, a cyst seems to develop and enlarge and if the hemorrhage occurs mainly within the angiomatous lesion, chronic encapsulated expanding hematoma seems to develop. Basically, cyst formation and chronic encapsulated expanding hematoma seem to be developed through the similar mechanism. In the irradiated field, hypoxic state may lead to the focal activation of the VEGF followed by neovascularization and these newly developed granulation tissues that can be fragile and continuous source of future bleedings may occur repeated hemorrhages, capsule formation, expansion of hematoma or cyst, and cerebral edema. Therefore, it may be reasonable to take radiological follow up images periodically for asymptomatic patients because of their possibility of progression of hematoma, cyst and cerebral edema by repeated minor hemorrhages. Symptomatic cysts can be indicated for Ommaya reservoir insertion to aspirate the contents repeatedly or cysto-peritoneal shunt (CPS). However, in the case of a large cyst greater than 70cc, stereotactic aspiration and CPS were performed after simple aspiration of cyst. One in six patients with cyst after radiosurgery for AVM has reported that despite stereotactic aspiration and CPS, a recurrence of the cyst formation has finally led to surgical resection ${ }^{15}$. The wide opening of cyst wall or removal of angiomatous lesion by craniotomy may be required ${ }^{815)}$. In some cases, monitoring devices are necessary to avoid any neurological impairment when performing surgery. The monitoring of visual field during surgical resection of a brain lesion was being conducted to reduce the occurrence of postoperative visual deficits. The intraoperative monitoring using flash visual evoked potentials was used as a detector of visual problems after surgery ${ }^{916)}$. According to the clinical experience from our case, excision or removal of lesion by craniotomy seems to be better than repeated aspirations if the lesion is enhanced on radiological images and accompanied by the repeated hemorrhages. Surgical resection by craniotomy has more advantages.

\section{CONCLUSIONS}

The angiomatous nodular lesions with cyst formation and repeated minor hemorrhages that developed in the adjacent cerebral parenchyma after SRS should be taken into consideration radiological follow-up and possibility of craniotomy for excision. Optimal treatment for symptomatic lesions seems to be excision of cyst including the associated angiomatous lesions.

\section{ACKNOWLEDGMENT}

Inje university Support for Academic Research Projects in 2011. 


\section{REFERENCES}

1. Ferrara N, Davis-Smyth TJEr. The biology of vascular endothelial growth factor. 1997;18(1):4-25.

2. Ferrara N, Gerber H-P, LeCouter JJNm. The biology of VEGF and its receptors. 2003;9(6):669.

3. Flickinger JC, Kondziolka D, Pollock BE, Maitz AH, Lunsford LDJIjoro, biology, physics. Complications from arteriovenous malformation radiosurgery: multivariate analysis and risk modeling. 1997;38(3):485-90.

4. Gökçil Z, Odabaşi Z, Atilla S, Kütükçü Y, Vural O, Yardim MJAnb. Radiological follow-up in encapsulated intracerebral hematoma mimicking intratumoural bleeding. 1998;98(1):27-31.

5. Greiner-Perth R, Neubauer U, Schenke HJNr. Chronic encapsulated intracerebral hematoma-a well-defined disease. Report on two cases and review of the literature. 1997;20(4):231-8.

6. Jain R, Robertson PL, Gandhi D, Gujar SK, Muraszko KM, Gebarski SJAJoN. Radiation-induced cavernomas of the brain. 2005;26(5):1158-62.

7. Kaido T, Hoshida T, Uranishi R, Akita N, Kotani A, Nishi N, et al. Radiosurgery-induced brain tumor: case report. 2001;95(4):710-3.

8. Kim M, Lee S, Sim J. A case of very large cyst formation with Gamma Knife radiosurgery for an arteriovenous malformation. Stereotactic and functional neurosurgery. 1999;72:168-74.

9. Kodama K, Goto T, Sato A, Sakai K, Tanaka Y, Hongo $\mathrm{K}$. Standard and limitation of intraoperative monitoring of the visual evoked potential. Acta neurochirurgica. 2010;152(4):643-8.

10. Kurita H, Sasaki T, Kawamoto S, Taniguchi M, Kitanaka $\mathrm{C}$, Nakaguchi $\mathrm{H}$, et al. Chronic encapsulated expanding hematoma in association with gamma knife stereotactic radiosurgery for a cerebral arteriovenous malformation: case report. 1996;84(5):874-8.

11. Marti HH, Risau WJPotNAoS. Systemic hypoxia changes the organ-specific distribution of vascular endothelial growth factor and its receptors. 1998;95(26):15809-14.
12. Marti HJ, Bernaudin $M$, Bellail A, Schoch $H$, Euler $M$ Petit E, et al. Hypoxia-induced vascular endothelial growth factor expression precedes neovascularization after cerebral ischemia. 2000;156(3):965-76.

13. Nagy G, Grainger A, Hodgson TJ, Rowe JG, Coley SC, Kemeny AA, et al. Staged-Volume Radiosurgery of Large Arteriovenous Malformations Improves Outcome by Reducing the Rate of Adverse Radiation Effects. Neurosurgery. 2017;80(2):180-92.

14. Nakamizo A, Suzuki SO, Saito N, Shono T, Matsumoto $\mathrm{K}$, Onaka S, et al. Clinicopathological study on chronic encapsulated expanding hematoma associated with incompletely obliterated AVM after stereotactic radiosurgery. 2011;153(4):883-93.

15. Pollock BE, Brown Jr RD. Management of cysts arising after radiosurgery to treat intracranial arteriovenous malformations. Neurosurgery. 2001;49(2):259-65.

16. Sasaki $T$, Itakura $T$, Suzuki $K$, Kasuya $H$, Munakata $R$, Muramatsu $\mathrm{H}$, et al. Intraoperative monitoring of visual evoked potential: introduction of a clinically useful method. Journal of neurosurgery. 2010;112(2):273-84.

17. Shuto T, Ohtake M, Matsunaga SJJon. Proposed mechanism for cyst formation and enlargement following Gamma Knife Surgery for arteriovenous malformations. 2012;117(Special_Suppl):135-43.

18. Takeuchi S, Takasato Y, Masaoka H, Hayakawa T, Otani $N$, Yoshino $Y$, et al. Development of chronic encapsulated intracerebral hematoma after radiosurgery for a cerebral arteriovenous malformation. 2009;151(11):1513.

19. Wang H-C, Chang RJ, Xiao FJSni. Hypofractionated stereotactic radiotherapy for large arteriovenous malformations. 2012;3(Suppl 2):S105.

20. Yamamoto $M$, Hara $M$, Ide $M$, Ono $Y$, Jimbo $M$, Saito IJSn. Radiation-related adverse effects observed on neuro-imaging several years after radiosurgery for cerebral arteriovenous malformations. 1998;49(4):385-98.

21. Yamamoto M, Jimbo M, Hara M, Saito I, Mori KJN. Gamma knife radiosurgery for arteriovenous malformations: long-term follow-up results focusing on complications occurring more than 5 years after irradiation. 1996;38(5):906-14. 